\title{
Avaliação dos efeitos do aspartame sobre a ingestão alimentar, os parâmetros físicos, bioquímicos e histopatológicos em ratos Wistar
}

[Evaluation of aspartame effects on food intake, physical, biochemical, and histopatological parameters in rats]

\author{
A.E. Silva ${ }^{1}$, M.A. Souza ${ }^{1}$, M.S.C. Gomes ${ }^{1}$, E.C.M. Souza ${ }^{1}$, M.F. Frazão ${ }^{1}$, C.G. D’assunção ${ }^{3}$, \\ C.S. Maia ${ }^{1}$, F.C.A.M. Tenório ${ }^{2 *}$, J.K.B. Soares ${ }^{1}$ \\ ${ }^{1}$ Universidade Federal de Campina Grande - Cuité, PB \\ ${ }^{2}$ Universidade Federal de Pernambuco - Recife, PE \\ ${ }^{3}$ Universidade Federal Rural de Pernambuco - Recife, PE
}

\begin{abstract}
RESUMO
Nos últimos anos, tem-se observado um aumento no consumo de alimentos diet e light por adolescentes ou por aqueles que estão à procura de uma alimentação com baixo teor calórico, surgindo, assim, diversos edulcorantes, como o aspartame. Porém, seu consumo ainda gera polêmica, devido a muitos dados contraditórios e inconclusivos. Diante disso, objetivou-se avaliar os efeitos da suplementação de aspartame sobre ingestão alimentar, parâmetros físicos, bioquímicos e histopatológicos em 18 ratos machos da linhagem Wistar, com cinco semanas de vida (ratos jovens), tratados durante 21 dias. Os animais foram distribuídos aleatoriamente em dois grupos: grupo controle (GC) - tratados com água destilada por gavagem, e o grupo aspartame (GA) - tratados diariamente com $2 \mathrm{~mL} / 100 \mathrm{~g} / \mathrm{dia}$ de aspartame. Todos os animais receberam ração comercial (Essence $\left.{ }^{\circledR}\right)$ e água ad libitum. O controle da ingestão alimentar foi registrado semanalmente. Foram aferidos os parâmetros físicos por meio da análise do peso corporal, da circunferência toráxica, da circunferência abdominal, do comprimento vérticecóccix, da gordura abdominal total e do cálculo do índice de massa corporal; os parâmetros bioquímicos foram analisados por meio da glicemia, da lipoproteína de alta densidade e dos triglicerídeos; além de tais análises, foi realizado o estudo histopatológico do fígado. Durante todo o experimento, os ratos tratados com aspartame apresentaram um aumento significativo no peso corpóreo e na ingestão alimentar quando comparados ao grupo controle. Não houve diferença nas demais análises tanto físicas, quanto bioquímicas e histopatológicas comparando-se o GA com o GC $(\mathrm{P}<0,05)$. Com base nos resultados obtidos, é possível inferir uma maior chance de desenvolvimento da obesidade, oriunda do consumo regular desse tipo de adoçante, já que ele comprovou ser capaz de estimular o consumo de alimentos e, consequentemente, o ganho de peso corpóreo.
\end{abstract}

Palavras-chaves: aspartame, peso corpóreo, ingestão alimentar

\begin{abstract}
Recent years have seen an increase in consumption of diet and light foods by teenagers or those who are looking for a low-calorie diet, thus resulting in several sweeteners such as aspartame. However, their consumption still generates controversy due to many contradictory and inconclusive data. Thus, the aim of this study was to evaluate the effects of aspartame supplementation on dietary intake, physical, biochemical, and histopathological parameters in 18 male Wistar rats, at five weeks old (young mice) treated for 21 days. The animals were randomly assigned into two groups: control group (CG) - treated with distilled water by gavage and aspartame group (GA) - treated with $2 \mathrm{ml} / 100 \mathrm{~g} /$ day of aspartame. All animals received commercial feed (Essence $\left.{ }^{\circledR}\right)$ and water ad libitum. The control of food intake was recorded weekly. The physical parameters were measured by analyzing the body weight, chest circumference, waist circumference, vertex-coccyx length, total abdominal fat and calculating the body
\end{abstract}

Recebido em 16 de junho de 2015

Aceito em 4 de abril de 2016

*Autor para correspondência (corresponding author)

E-mail: fcas14@hotmail.com 
mass index; biochemical parameters were analyzed by glucose, high-density lipoprotein and triglycerides. Apart from such analysis the histopathological study of the liver was conducted. Throughout the experiment the rats treated with aspartame showed a significant increase in body weight and food intake compared to the control group. There was no difference in other analyzes such physical, biochemical, and histopathological comparing GA to GC $(P<0.05)$. From the results we can infer a greater chance of developing obesity, coming from the regular consumption of this type of sweetener, as it proved able to stimulate food intake, and hence the gain of body weight.

Keywords: Aspartame, body weight, food intake

\section{INTRODUÇÃO}

Nos últimos anos, o consumo de alimentos diet e light tem aumentado constantemente pela população em geral e tem estimulado um maior investimento na elaboração de novos produtos direcionados a indivíduos que apresentam algum distúrbio no metabolismo de açúcares (diabéticos) ou, mais recentemente, a consumidores que estão à procura de alimentos com baixo teor calórico. Existem diversos edulcorantes permitidos para uso em alimentos e bebidas no Brasil, dentre os quais, os mais vendidos são o ciclamato de sódio, a mistura de sacarina e o aspartame (Freitas e Araújo, 2010).

O aspartame, ou éster metílico de N-alfaaspartil-L-fenilalanina, foi descoberto em 1965 por James Schlatter (Roberts, 1996). Porém, seu consumo só foi liberado pelo Food and Drug Administration (FDA) - órgão norte-americano responsável por testar drogas, alimentos e cosméticos - em 1981, após a realização de diversos estudos toxicológicos (Martins e Azoubel, 2006). No Brasil, o livre comércio de dietéticos foi autorizado em 1988 (Martins e Azoubel, 2006).

Todavia, a ingestão do aspartame ainda gera polêmica, devido a muitos dados contraditórios e inconclusivos sobre o seu consumo como aditivo alimentar, principalmente em relação ao peso corpóreo, à ingestão alimentar e à saciedade (Reis, 2010). Entre essas modificações, pode-se citar o estudo realizado por Souza et al. (2010), o qual utilizou ratas do oitavo ao $12^{\circ}$ dia de prenhez e verificou que o grupo tratado com aspartame apresentou maiores taxas de glicose, colesterol total, lipoproteína de alta densidade (HDL), maior peso corpóreo e más-formações das proles. Em contrapartida, em outro estudo, realizado por Vieira et al. (2008), uma solução de aspartame a
$1 \%$ reduziu os níveis de triglicerídeos em ratos adultos tratados.

Estudos também afirmam que o aspartame pode ser usado para o controle da obesidade e do diabetes mellitus (Araújo et al., 2008). Contudo, assegura-se que ele pode estimular o apetite paradoxalmente e, assim, levar ao ganho de peso corporal (Anton et al., 2010).

Pelo fato de os adolescentes consumirem grandes quantidades de produtos contendo esse edulcorante, chegando a ingerir o dobro de aspartame comparado ao consumo de adultos e idosos, e por seu organismo estar ainda em formação, a consequência bioquímica e histopatológica do consumo exacerbado desse edulcorante é de suma importância para o conhecimento dos profissionais de saúde que buscam maiores informações sobre os benefícios e malefícios desse alimento, uma vez que ainda são observadas diversas controvérsias decorrentes de seu consumo.

\section{MATERIAL E MÉTODOS}

Foram utilizados 18 ratos machos, adolescentes, da linhagem Wistar, provenientes do Laboratório de Nutrição Experimental da Universidade Federal de Campina Grande- PB, com cinco semanas de vida, pesando aproximadamente $108 \mathrm{~g}$. O experimento foi aprovado pela Comissão de Ética Institucional: CEP- $\mathrm{N}^{\circ} 128-2013$.

Os animais foram alocados aleatoriamente em dois grupos de nove animais cada:

grupo controle (GC) - tratados com água destilada por via orogástrica;

grupo Aspartame (GA) - tratados com aspartame, uma vez ao dia, por via orogástrica, sendo administrados $2 \mathrm{~mL} / 100 \mathrm{~g} /$ dia durante 21 
dias. Todos os animais receberam ração padrão (Essence $\left.{ }^{\circledR}\right)$ e água ad libitum. Durante todo o experimento, os animais foram mantidos em gaiolas metabólicas individuais, em temperatura entre $22 \pm 3^{\circ} \mathrm{C}$ e umidade relativa do ar de $60 \pm 3 \%$, ciclo de $12 \mathrm{~h}$ claro/escuro.

O aspartame utilizado na referida pesquisa foi adquirido em um comércio local de Cuité. Apresentava disponível na forma em pó, da marca Finn ${ }^{\circledR}$. Ele, então, foi diluído para ser administrado por via orogástrica, na dosagem de $2 \mathrm{~mL} / 100 \mathrm{~g} /$ dia, durante 21 dias.

O controle da ingestão alimentar foi realizado semanalmente em balança semianalítica da marca Balmax (modelo: ELP - 25).

As medidas murinométricas foram realizadas em todos os ratos, momentos antes da eutanásia, quando estes foram anestesiados com cloridrato de ketamina e xilazina $(1 \mathrm{~mL} / \mathrm{kg})$. Foi mensurada a circunferência abdominal (CA), a circunferência toráxica (CT) e o comprimento do corpo. O ganho de peso total foi definido pela subtração entre o peso inicial, ou seja, antes dos respectivos tratamentos, e o peso final, expresso em gramas. Para aferição do peso corporal, foi utilizada uma balança semianalítica da marca Balmax, modelo: ELP - 25 (Novelli et al., 2007).

O peso corporal e o comprimento do corpo foram usados para determinar o índice de massa corporal $(\mathrm{IMC})=\mathrm{de}$ peso corporal $(\mathrm{g}) /$ comprimento $\left(\mathrm{cm}^{2}\right)$.

A gordura abdominal total foi retirada e pesada em uma balança semianalítica da marca Balmax, modelo: ELP - 25 .

Para coleta de sangue, os animais permaneceram em jejum de 12 horas. As amostras foram coletadas por meio da punção cardíaca ao final do experimento. Os níveis de glicose plasmática foram determinados utilizando-se um glicosímetro ACCU-CHEK $®$.

Para as análises de triglicerídeos e HDL, as amostras foram centrifugadas a 3000rpm, durante $10 \mathrm{~min}$, a $4^{\circ} \mathrm{C}$, para a obtenção do soro. Foi, então, utilizado kit enzimático (Labtest).
A coleta do fígado ocorreu no momento da eutanásia, que se deu mediante o aprofundamento anestésico com ketamina e xilazina. A secção tecidual foi fixada em formalina tamponada $10 \%$ e, após 24 horas, resseccionada para o processamento histopatológico: desidratação com séries crescentes de álcool (70\% a 100\%), seguida de diafanização em xilol, impregnação e inclusão em parafina, segundo os métodos de rotina (Michalany, 1998). Posteriormente, os blocos de parafina foram cortados em micrótomo ajustado para $3,0 \mu \mathrm{M}$. Os cortes obtidos foram colocados em lâminas untadas com albumina de Meyer e submetidos à coloração com hematoxilinaeosina, as quais, em seguida, foram analisadas em microscópio de luz.

Os resultados das análises foram submetidos ao teste t-Student, seguido de Mann-Whitney, com nível de significância $\mathrm{P} \leq 0,05$.

\section{RESULTADOS}

Com relação ao consumo de ração, o $\mathrm{GC}$ comparado com o GA, respectivamente, apresentou: primeira semana $(150 \pm 8,94 \mathrm{x}$ $160,67 \pm 9,17)$, segunda semana $(151,71 \pm 15,51$ $\mathrm{x} 164,22 \pm 15,01)$, terceira semana $(158,67 \pm$ $13,95 \times 185,71 \pm 23,02)$ (Fig.1).

De acordo com os dados, pode-se verificar que os ratos tratados com aspartame (GA) apresentaram um aumento significativo na ingestão alimentar na primeira, segunda $\mathrm{e}$ terceira semanas, quando comparados ao grupo controle $(\mathrm{GC}),(\mathrm{P} \leq 0,05)$.

Quanto ao ganho de peso corpóreo, o GC confrontado com o GA, respectivamente, apresentou menores valores significativamente durante a primeira semana $(139,25 \pm 13,48 \mathrm{x}$ $150 \pm 13,96)$, segunda semana $(168,75 \pm 20,37 x$ $192 \pm 17,53)$ e terceira semana $(204,86 \pm 18,97$ $\mathrm{x} 228,29 \pm 17,18)(\mathrm{P} \leq 0,05)($ Fig. 2$)$.

Com relação às medidas murinométricas circunferência abdominal, circunferência toráxica, comprimento, gordura abdominal total e IMC, podem-se observar, tanto no GC $(n=9)$ quanto no GA $(n=9)$, resultados semelhantes $(\mathrm{P} \leq 0,05)$ (Tab. 1). 


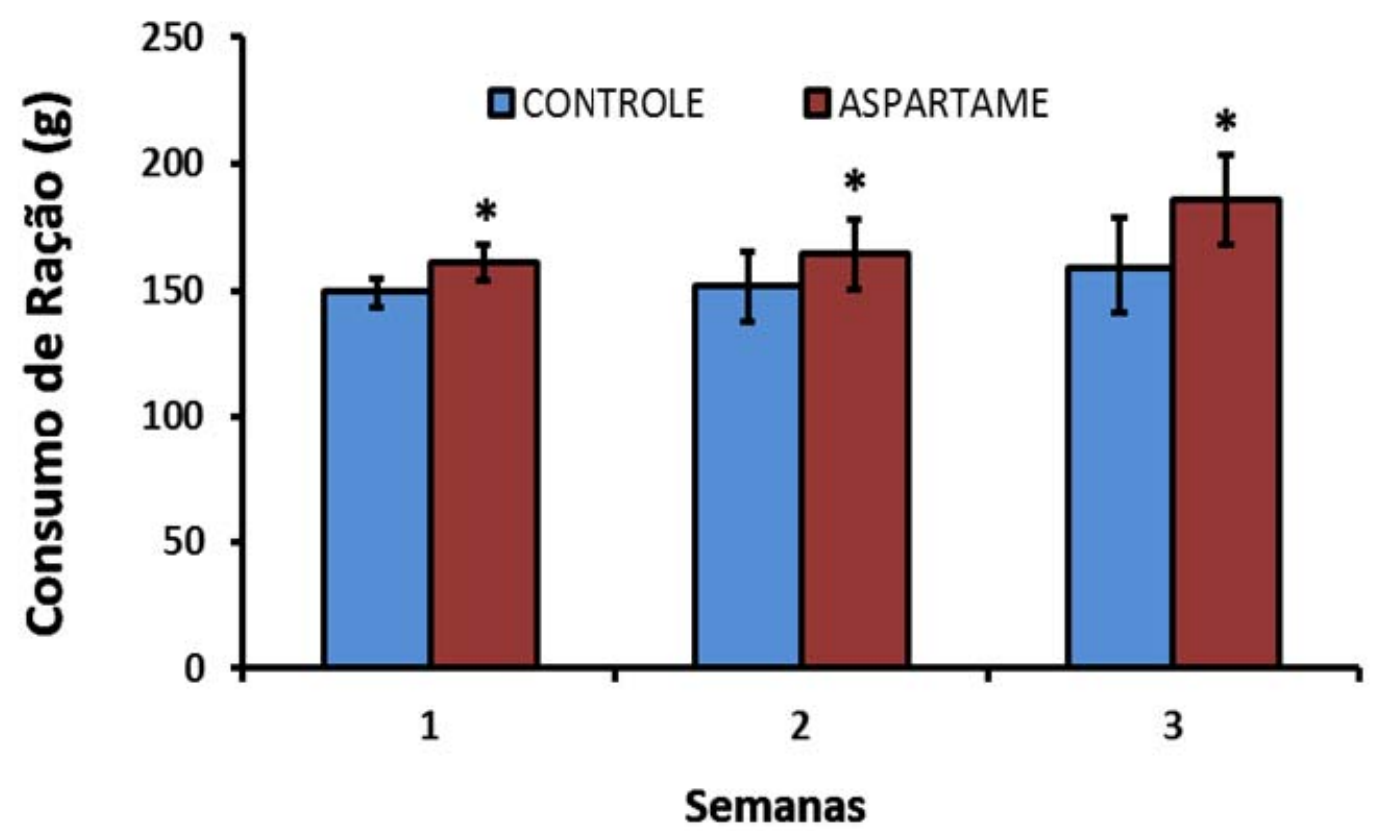

Figura 1. Consumo de ração comercial (Essence $($ ) ) de ratos jovens da linhagem Wistar, tratados por gavagem durante 21 dias, quando GA recebeu aspartame a 5\% $(\mathrm{n}=9)$ e o $\mathrm{GC}$ recebeu solução de água destilada $(\mathrm{n}=9)$. Dados expressos em média \pm DP. Foram aplicados os testes t-Student e Mann-Whitney para se verificarem diferenças estatísticas. *= diferença GA vs. $\mathrm{GC}(\mathrm{P} \leq 0,05)$.

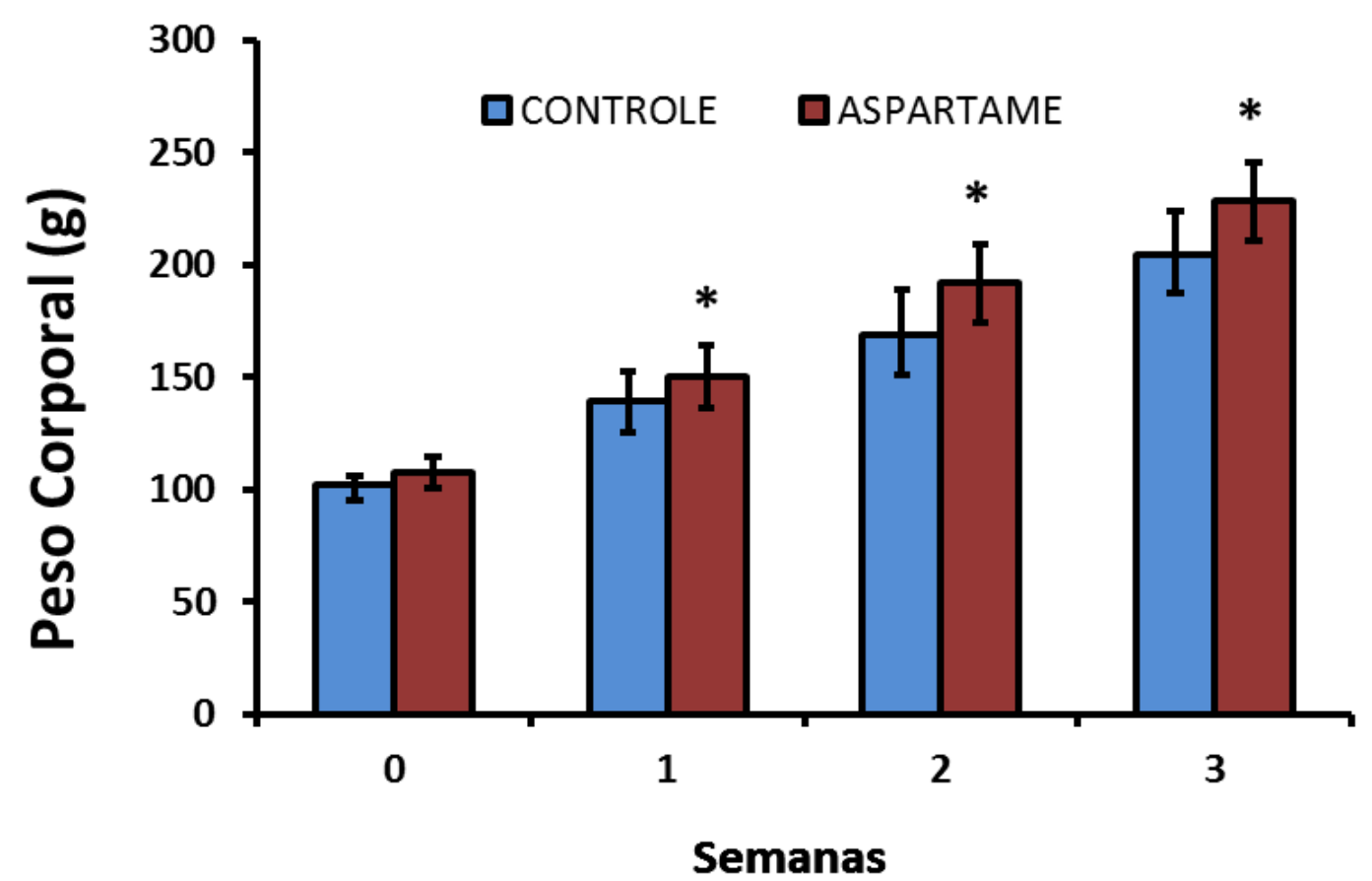

Figura 2. Evolução do ganho de peso de ratos adolescentes Wistar alimentados durante 21 dias com ração

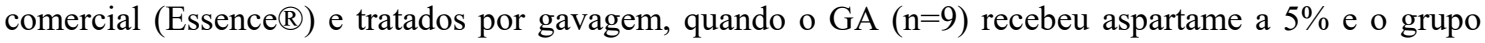
controle $(\mathrm{GC})(\mathrm{n}=9)$ recebeu água destilada. Dados expressos em média \pm DP. Foram aplicados os testes t-Student e Mann-Whitney para se verificarem diferenças estatísticas. *=diferença significativa comparado ao GC. 


\section{Silva et al.}

Tabela 1. Medidas murinométricas de ratos adolescentes da linhagem Wistar tratados durante 21 dias por gavagem, quando o GA recebeu solução a $5 \%$ de aspartame $(n=9)$ e o grupo controle $(n=9)$ recebeu água destilada

\begin{tabular}{lcc}
\hline \multirow{2}{*}{ Medidas murinométricas } & \multicolumn{2}{c}{ Grupos experimentais } \\
\cline { 2 - 3 } & Controle $(\mathrm{n}=9)$ & Aspartame $(\mathrm{n}=9)$ \\
\hline Circunferência abdominal $(\mathrm{cm})$ & $13,50 \pm 1,25$ & $14 \pm 1,36$ \\
Circunferência toráxica $(\mathrm{cm})$ & $12 \pm 0,71$ & $12 \pm 1,03$ \\
Comprimento $(\mathrm{cm})$ & $21,25 \pm 1,04$ & $21,50 \pm 0,71$ \\
Gordura abdominal total $(\mathrm{g})$ & $4,54 \pm 1,87$ & $5,52 \pm 1,50$ \\
$\mathrm{IMC}\left(\mathrm{g} / \mathrm{cm}^{2}\right)$ & $0,50 \pm 0,02$ & $0,46 \pm 0,06$ \\
\hline
\end{tabular}

Fonte: Dados expressos em média \pm DP. Utilizou-se para análise dos dados o teste t-Student, seguido de MannWhitney $(\mathrm{P} \leq 0,05)$.

No que se refere aos dados bioquímicos, pode-se notar que o GA e o GC apresentaram resultados semelhantes em relação à glicemia, ao triglicerídeo e ao HDL $(\mathrm{P} \leq 0,05)$ (Tab. 2).

Os resultados mostraram que os fígados de todos os grupos experimentais apresentaram externamente uma delgada cápsula composta de tecido conjuntivo fibroso, a cápsula de Glisson
(Fig. 3A), um parênquima bem desenvolvido e com uma malha vascular com veias de vários calibres (Fig. 3B). Os hepatócitos dispuseram-se desordenadamente em torno da veia centro-lobular, margeando os capilares sinusoides (Fig. 3C). Essas células apresentaram morfologia poliédrica, núcleo central, nucléolo proeminente e citoplasma acidófilo (Fig. 3D).

Tabela 2. Dados bioquímicos de ratos Wistar tratados durante a adolescência com aspartame a 5\% (GA) e água destilada (GC)

\begin{tabular}{lll}
\hline \multirow{2}{*}{ Parâmetros bioquímicos } & Grupos experimentais & \\
\cline { 2 - 3 } & Controle $(\mathrm{n}=9)$ & Aspartame $(\mathrm{n}=9)$ \\
\hline Glicemia $(\mathrm{mg} / \mathrm{dL})$ & $170,88 \pm 35,47$ & $178 \pm 39,79$ \\
Triglicerídeos $(\mathrm{mg} / \mathrm{dL})$ & $28,99 \pm 9,72$ & $31,61 \pm 14,92$ \\
HDL $(\mathrm{mg} / \mathrm{dL})$ & $57,55 \pm 2,34$ & $53,01 \pm 3,72$ \\
\hline
\end{tabular}

Fonte: Dados expressos em média \pm DP. Utilizou-se para análise dos dados o teste t-Student, seguido de MannWhitney. *Indica diferença em relação ao $\mathrm{GC}(\mathrm{P} \leq 0,05)$.
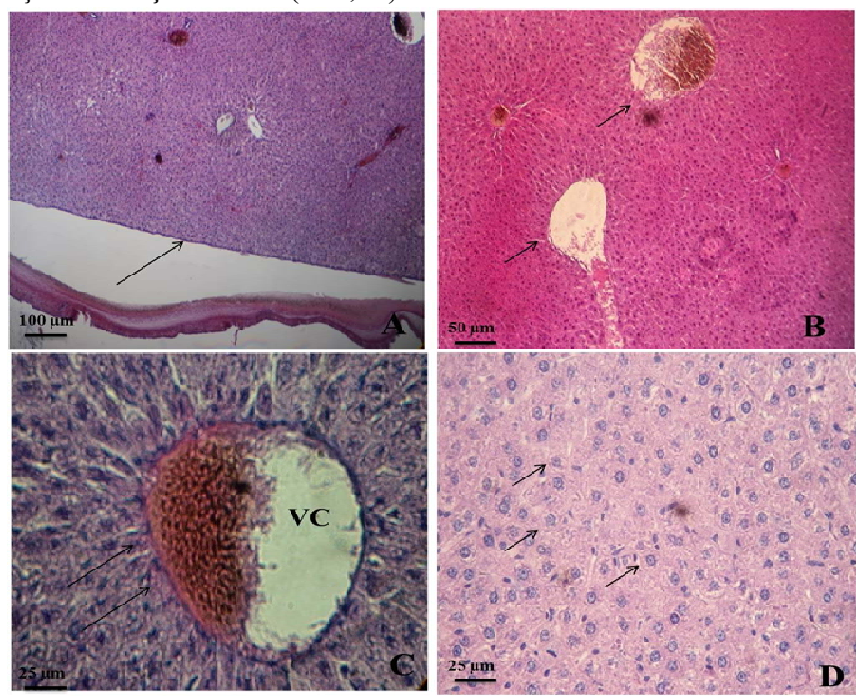

Figura 3. Histologia do fígado de ratos dos grupos experimentais. A - cápsula de Glisson (seta) do grupo controle; B - veias de vários calibres (seta pequena) no grupo aspartame; C - hepatócitos (setas) em torno da veia centro-lobular (VC) no grupo aspartame; D - hepatócitos (setas pequenas) com morfologia poliédrica, núcleo central, nucléolo proeminente e citoplasma acidófilo do grupo aspartame. Coloração HE. 


\section{DISCUSSÃO}

Com relação ao peso corporal, os dados da presente pesquisa corroboram trabalhos que utilizaram animais tratados com aspartame em diferentes concentrações e em diferentes fases da vida. Estudo que trataram ratas prenhes com $14 \mathrm{mg} / \mathrm{kg}$ de aspartame identificaram um aumento no peso materno e no peso placentário (Leme e Azoubel, 2006).

Souza et al. (2010) observaram maior peso corporal na prole de ratas tratadas com $25 \mathrm{mg} / \mathrm{kg}$, e Luz (2008) identificou o mesmo aumento de peso em ratos adultos que receberam 20 gotas $/ 500 \mathrm{~mL}$ de adoçante artificial. Em ambos os estudos, o maior consumo de ração estava presente, o que leva a inferir que o aspartame estimula o apetite e é esse aumento que induz ao ganho de peso. Esse aumento de apetite já havia sido relatado por outros autores (Rosado e Monteiro, 2001; Anton et al., 2010). Wurtman (1983) sugere que esse aumento do apetite se deve à redução das concentrações de serotonina na presença do aspartame, já que a serotonina age regulando a sensação de saciedade e a sua redução resulta no aumento da fome.

No que se refere aos parâmetros físicos, bioquímicos e histológicos analisados, na atual pesquisa, não houve alterações no grupo tratado com aspartame em comparação ao controle. Porém, quanto a este último, dados corroboram o estudo que utilizou esse mesmo adoçante, no qual ratos que foram tratados com $1 \%$ de aspartame por cinco meses não apresentaram nenhuma alteração no fígado, no duodeno, no baço, na bexiga e no pâncreas quando comparados com animais controle (Vieira et al., 2008). No entanto, o achado de Abhilash et al. (2011) difere do resultado supracitado. Em ratos adultos tratados com uma elevada concentração $(1000 \mathrm{mg} / \mathrm{kg})$ durante 180 dias, esses autores observaram um aumento significativo nas atividades das enzimas séricas alanina aminotransferase (ALT), aspartato aminotransferase (AST), fosfatase alcalina (ALP) e c-glutamil transferase (GGT), aumento esse que pode indicar lesões hepáticas. Houve, ainda, redução na concentração de glutationa reduzida (GSH), na atividade da glutationa peroxidase (GPx), da glutationa redutase (GR) e infiltração de leucócitos nos fígados dos ratos. Esses achados indicam uma toxicidade do aspartame. Com base no exposto, tanto a dosagem quanto o período de administração foram capazes de alterar essa toxicidade.

Pesquisas com animais de laboratório sugerem que jovens que consomem esse tipo de adoçante regularmente possuem maiores chances de desenvolver obesidade, já que ele estimula o consumo de alimentos, proporcionando um aumento na ingestão alimentar e, consequentemente, no ganho de peso corpóreo, o que pode contribuir posteriormente para o aparecimento de doenças crônicas. Dessa forma, o risco que os adolescentes correm ao consumir esse edulcorante, ou produtos que o contenham, é consideravél, uma vez que, segundo Branco et al. (2006), eles são os maiores consumidores dos produtos diéteticos. Pelo fato de, nessa faixa etária, o organismo ainda estar em fase de formação, a consequência do seu consumo exacerbado por adolescentes pode ser deletéria ao organismo, sendo alguns desses riscos confirmados na presente pesquisa.

\section{CONCLUSÃO}

Foi possível concluir que o aspartame causou um aumento significativo tanto na ingestão alimentar quanto no peso corpóreo em ratos jovens; no entanto, não houve alterações nos parâmetros físicos, bioquímicos e histopatológicos analisados, fazendo com que esse edulcorante não possa ser considerado a melhor opção para substituição do açúcar por indivíduos que almejam uma redução calórica. Porém, apresentou resultados benéficos para o controle da glicemia, uma vez que os ratos que ingeriram aspartame não apresentaram alterações nos níveis de glicose, sendo uma excelente alternativa de substituição do açúcar por pessoas que buscam o controle dos níveis glicêmicos.

\section{REFERÊNCIAS}

ABHILASH, M.; SAUGANTH, P.; VARGHESE, M.V.; NAIR, R.H. Effect of long term intake of aspartame on antioxidant defense status in liver. Mag. Elsevier, v.49, p.1203-1207, 2011.

ANTON, S.D.; MARTIN, C.K.; HAN, H. et al. Effects of stevia, aspartame, and sucrose on food intake, satiety, and postprandial glucose and insulin levels. Appetite, v.55, p.37-43, 2010. 
ARAÚJO, D.B.; BARRAL, T.; ARAÚJO, R.P.C. Análise das características de produtos contendo aspartame comercializados em Salvador, Bahia, Brasil. Pesqui. Bras. Odontopediatr. Clín. Integr., v.8, p.223-228, 2008.

BRANCO, L.M.; HILÁRIO, M.O.E.; CINTRA, I.P. Relação entre o consumo de alimentos dietéticos e light com a condição nutricional, a percepção e a satisfação da imagem corporal em adolescentes. $J$. Bras. Soc. Nutr., v.31, p.27-36, 2006.

FREITAS, A.S.; ARAÚJO, A.B. Edulcorante artificial: aspartame - uma revisão de literatura. Rev. Eletron. Multidisc. Pindorama IFBA, v.1, p.1-11, 2010.

LEME， L.F.A.G.; AZOUBEL， R. Effects of aspartame on the exocrine pancreas of rat fetuses. Int. J. Morphol., v.4, p.679-684, 2006.

LUZ, B.S. Avaliação fisiológica em ratos wistar na ingestão de açúcar e adoçante artificial. 2008. 16f. TCC (Trabalho de Conclusão do Curso de Nutrição) Faculdade Assis Gurgacz, Cascavel, PR.

MARTINS, M.R.I.; AZOUBEL, R. Efeitos do aspartame no rim fetal de ratos - estudo cariométrico. J. Bras. Nefrol., v.28, p.151-157, 2006

MICHALANY, J. Técnica histológica em anatomia patológica: com instruções para o cirurgião, enfermeira e citotécnico. 3.ed. São Paulo: Michalany, 1998. 295p.
NOVELLI, E.L.B.; DINIZ, Y.S.; GALHARDI, C.M et al. Anthropometrical parameters and markers of obesity in rats. Lab. Anim., v.41, p.111-119, 2007.

REIS, C. Efeitos do adoçante dietético (aspartame) e da sacarose no peso corporal e na ingestão calórica de ratos wistar. 2010. 84f. Dissertação (Mestrado em ciências médicas) - Faculdade de Medicina, Universidade Federal do Rio Grande do Sul, RS.

ROBERTS, H.J. Aspartame as a cause of allergic reactions, including anaphylaxis. Arch. Intern. Med., v.156, p.156-167, 1996.

ROSADO, E.L.; MONTEIRO J.R. Obesidade e a substituição de macronutrientes da dieta. Rev. Nutr., v.14, p.145-52, 2001

SOUZA, M.L.G.V.; COSTA, A.M.D.D.; TERRA, F.S.; SOUZA, F.V. Avaliação do efeito do aspartame em ratas tratadas durante a prenhez e o reflexo do tratamento em seus fetos. Rev. Bras. Clín. Méd., v.8, p.328-332, 2010.

VIEIRA, C.A.; CORBO, M.A.P.; BORGES, T.M. et al. Adoçantes dietéticos na alimentação de ratos: uma abordagem histológica e bioquímica. Semin. Ciênc. Biol. Saúde, v.29, p.105-114, 2008.

WURTMAN, R.J. Neurochemical changes following high-dose aspartame with dietary carbohydrate. $N$. Engl. J. Med., v.309, p.429-430, 1983. 